\title{
Potência de um ponto: Provas Matemática e Física
}

\author{
Yuri M. Rodella ${ }^{1}$ \\ ProfMat/UFSCar, São Carlos, SP \\ J. A. Salvador ${ }^{2}$ \\ DM/UFSCar, São Carlos, SP
}

\begin{abstract}
Resumo. A demonstração matemática é um procedimento de validação de um resultado que embasa uma proposição de forma a garantir a sua veracidade e ela está no cerne de todo o pensamento matemático. Uma demonstração matemática pode parecer abstrata e difícil para a maioria dos estudantes do Ensino Básico. Na busca de diminuir esta dificuldade, propomos explorar a interdisciplinaridade resgatando a demonstração de teoremas matemáticos sobre a potência de um ponto em relação a uma circunferência com base em argumentações físicas, uma vez que força e pressão são palavras mais presentes na vida prática dos estudantes do que congruência de triângulos.
\end{abstract}

Palavras-chave. Potência de um ponto; Interdisciplinaridade; Geometria; Demonstrações Físicas.

\section{Introdução}

Como a Física e a Matemática procuram descrever o universo que nos rodeia, cada uma com suas características próprias, deve existir alguma equivalência entre essas duas linguagens que pode ser usada nas demonstrações de resultados matemáticos. E, nos últimos anos, despontaram propostas para o Ensino Básico voltadas para o desenvolvimento de habilidades e competências, adoção de eixos norteadores, ensino transversalizado ou ainda a de temas geradores de forma a direcionar um conjunto de disciplinas como um todo apontando para o avanço da interdisciplinaridade na sala de aula, como indica o Currículo do Estado de São Paulo [9].

As ideias aqui apresentadas sobre a integração da Física com a Matemática básicas, poderão servir de incentivo e motivação para esta área que, embora desejada, carece de bons materiais de referência sobre modelos de interdisciplinaridade.

Na Matemática, o uso de registros de representação e das diferentes linguagens é de grande importância para a compreensão, resolução e comunicação de resultados de uma atividade [6]. Ainda, o trânsito entre os diversos registros de representação pode favorecer os estudantes a adquirirem maior flexibilidade e fluidez nas diversas áreas da ciência, desenvolvendo o raciocínio e incrementando a conexão entre elas.

O uso de argumentos físicos neste trabalho propõe a construção de uma dinâmica interdisciplinar sobre como fazer uma demonstração, passo a passo, com sutilezas físicas de uma maneira mais perceptível ou visual, tomando por base conceitos presentes no dia a dia do estudante. Demonstrações diferenciadas ainda são discutidas por Nasser e Tinoco [7] e defendidas por Balacheff [1].

Apresentaremos alguns resultados sobre a potência de um ponto usando conceitos básicos da teoria dos gases. Ressaltamos que Arquimedes de Siracusa (287 a.C. - 212 a.C.) fez grandes descobertas matemáticas, fundamentando sua busca em ideias físicas, como as do equilíbrio entre braços de alavancas e da hidrostática [5], e também em Rodella e Salvador [8] onde apresentamos uma

\footnotetext{
1yuridi@gmail.com

${ }^{2}$ salvador@dm.ufscar.br
} 
demonstração física do teorema de Pitágoras para estudantes do Ensino Básico usando conceitos de hidrostática.

\section{Potência de um ponto em relação a uma circunferência}

A potência de um ponto em relação a uma circunferência é de grande importância em várias aplicações geométricas, especialmente na resolução de problemas interessantes envolvendo relações métricas de secantes e tangentes a uma circunferência, embora parece esquecida na maioria dos currículos e dos livros do Ensino Básico.

A potência de um ponto $P$ a uma distância $d$ da circunferência de centro $O$ e raio $r$, é definido como

$$
\operatorname{Pot}(P)=d^{2}-r^{2}
$$

Esta definição é equivalente a propriedade de que se uma reta passa por um ponto $P$ e corta a circunferência nos pontos $A$ e $B$ conforme a Figura 1, então o produto $P A \cdot P B$ é constante [10].
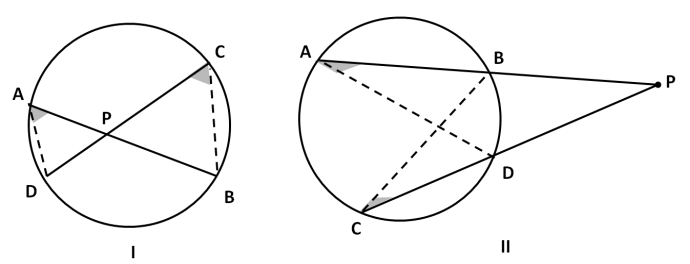

Figura 1: Potência de um ponto P.

As demonstrações geométricas muitas vezes descrevem como as proporções das medidas de certos comprimentos de objetos se relacionam entre si, ou mesmo como as de algumas áreas ou volumes se relacionam com outras.

Discutiremos aqui, uma técnica demonstrativa de como princípios físicos simples pode nos levar às mesmas relações matemáticas, e o que permite esta adaptação, é a possibilidade de se intercambiar o comprimento de objetos geométricos pela força atuante em objetos físicos, que guardam estreita relação com as propriedades dos objetos geométricos abordados no Ensino Médio refletindo alguns aspectos da realidade.

Na maioria das vezes trabalhamos geometricamente com objetos estáticos, e portanto, a combinação de forças atuantes sobre eles devem obedecer uma condição de equilíbrio material: o somatório das forças ou o somatório dos torques resultantes devem ser nulos.

Vamos estabelecer um resultado que caracteriza a potência de um ponto em relação a uma circunferência interdisciplinarmente e ressaltar os principais conceitos matemáticos e físicos utilizados em cada um dos casos. 


\subsection{Teorema da Potência de um Ponto}

Observamos que o conceito de potência de um ponto em relação a uma circunferência se aplica para um ponto $P$ interior ou exterior da circunferência $C$ conforme mostrado na Figura 1.

a) Ponto Interior a uma Circunferência: Se duas cordas $\overline{A B}$ e $\overline{C D}$ de uma mesma circunferência se interceptam num ponto $P$, então o produto das medidas das duas partes de uma é igual ao produto das medidas das duas partes da outra.

b) Ponto Exterior a uma Circunferência: Se por um ponto exterior $P$ a uma circunferência, traçarmos duas secantes $\overline{P A}$ e $\overline{P B}$ a ela, então o produto da medida da primeira secante pela sua parte exterior é igual ao produto da medida da segunda secante pela sua parte exterior.

\section{Demonstração Matemática:}

A demonstração Matemática para o Teorema da Potência de um Ponto, em relação a uma dada circunferência, é relativamente simples e se faz por semelhança de triângulos.

Observando a Figura 1-I e 1-II, temos que em ambos os casos distintos, o arco $\overparen{\mathrm{DB}}$ das circunferências é comum tanto ao ângulo $\hat{A}$ quanto ao ângulo $\hat{C}$ que são congruentes.

Observamos que, para os triângulos $\triangle A D P$ e $\triangle B C P$, o ângulo marcado por $\hat{P}$ tem medidas iguais em cada uma das figuras 1-I e 1-II. Na Figura 1-I eles são opostos pelo vértice e, na 1-II, $\hat{P}$ é um ângulo comum aos dois triângulos semelhantes mencionados, assim a seguinte relação pode ser descrita:

$$
\frac{P A}{P C}=\frac{P D}{P B} \Longleftrightarrow P A \cdot P B=P C \cdot P D
$$

Esta demonstração é simples e abstrata. Simples por se tratar de uma aplicação pura e direta da semelhança de triângulos, mas a mesma sequência de passos pode ser utilizada tanto para a Figura 1-I, quanto para a Figura 1-II, as quais são muito diferentes.

O desafio do professor é desencadear todo um conjunto de premissas básicas de forma a compor uma argumentação sólida e incontestável para que o estudante possa adquirir o raciocínio lógico e abstrato, ou seja, como se construir uma demonstração.

\section{Demonstração Física:}

Fisicamente o conceito de pressão, mesmo que ainda não formalizado de maneira sistemática pela escola, é algo que está bastante presente no cotidiano do estudante. Seja nos pneus dos carros ou das bicicletas, seja nas boias de piscina, o conceito do dia a dia de pressão é de fácil percepção para um estudante, ainda que a sua descrição física seja excepcionalmente abstrata.

Também o princípio das alavancas, o qual assegura que se uma gangorra de braços de tamanho desiguais está em equilíbrio, o produto do comprimento dos braços de apoio, em relação ao fulcro, pelos seus respectivos pesos suportados deve sempre se igualar.

Imaginemos, pois, uma caixa hermeticamente fechada, com vista superior como mostrado na Figura 2 e com paredes laterais de altura unitária, onde o compartimento ATP destacado tem um gás aprisionado.

Obviamente que ao se encher uma caixa com gás comprimido, esta ação não vai fazer com que ela saia se movimentando desgovernadamente, ela continuará em repouso. Entretanto, suas paredes devem suportar forças que não existiam anteriormente, quando ela se encontrava vazia. A pressão do gás no interior da Figura 2, de vértices $A, T$ e $P$, faz com que suas paredes $\overline{A T}$, TP e $\overline{A P}$ tendam a se arrebentar, formando um embarrigamento, vista do interior do recipiente, tenda para o formato convexo, entretanto, se a mesma for resistente ela manterá o formato.

Mensurando o valor das forças atuando sobre as faces do invólucro, verificamos que elas são diretamente proporcionais aos comprimentos dos respectivos lados sobre as quais atuam, devido a altura unitária dos lados. 


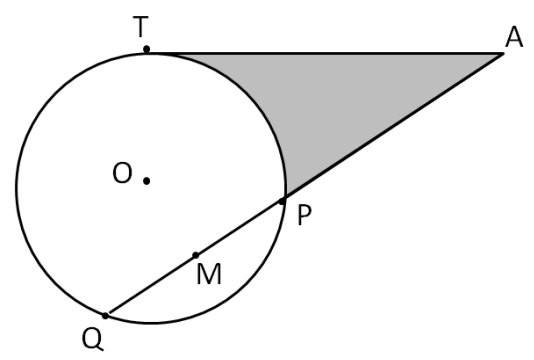

Figura 2: Demonstração física para a potência de um ponto.

A prova disso é que, se a pressão $p$ é definida como a relação de força $F_{p}$ sobre uma determinada área $A$,

$$
p=\frac{F_{p}}{A},
$$

segue que a força $F_{p}$ que atua sobre cada face é dada pela pressão interna $p$ do gás multiplicada pela área $A$, que por sua vez, é o comprimento da face $c$ multiplicado pela altura $h$, ou seja,

$$
F_{p}=p \cdot A=p c h,
$$

Tendo o recipiente uma altura $h$ unitária (ou constante), a área da parede $A T$, por exemplo, equivale ao comprimento da própria parede $A T$, ajustando-se a pressão $p$ também para uma pressão unitária, teremos a relação de $F_{A T}=A T$, e da mesma forma para os outros lados, ou seja, $F_{A P}=A P$.

É evidente que o mesmo ocorre para a superfície curva definida por $\overparen{\mathrm{TP}}$, entretanto, aqui usamos o centro da circunferência da Figura 3 como referência para o cálculo dos torques gerados por todas as forças de pressão. Dado que a força de pressão, resultante em $\overparen{\mathrm{TP}}$, aponta em direção ao ponto central $O$, não causa uma tendência de giro em relação a ele, não precisando ser determinada, de forma que, em relação a $O$, apenas as forças $F_{A T}$ e $F_{A P}$ devem ser equilibradas.

Sabe-se que o somatório dos torques de todas as forças que atuam sobre um objeto em repouso, com base em qualquer ponto de referência, é nulo, e estabelecendo-se como ponto de referência o centro $O$ da circunferência, a adoção de tal referencial simplificará muito os cálculos. Um vez que a força $F_{A T}$ impõe uma tendência de giro no ponto $\mathrm{O}$ de sentido anti-horário, o torque causado por esta força é negativo e, da mesma maneira, a força $F_{A P}$ causa uma tendência de giro sobre o ponto $O$ de sentido horário, de forma que o torque gerado por esta força terá sinal positivo. Notamos também o sentido da convenção de sinais.

Observamos na Figura 3-II que a distância da linha de ação da força $F_{A T}$ ao ponto $O$ é exatamente a metade da distância $A T$, de forma que toda a dificuldade deste cálculo se resume tão somente em se determinar a distância da linha de ação da força $F_{A P}$ ao ponto $O$.

Percebemos pela Figura 3-I que a distância do ponto $O$ à reta suporte desta é igual à distância do ponto médio do segmento $\overline{A P}$ ao ponto médio $M$ da corda $\overline{P Q}$, pois a reta suporte da força $F_{A P}$ é paralela ao segmento $\overline{O M}$, uma vez que ambas são perpendiculares a um mesmo segmento de reta $\overline{A Q}$

Assim, a distância do ponto de aplicação da força $F_{A P}$ ao ponto $M$ é dado por

$$
P M+\frac{A P}{2}=\frac{P Q}{2}+\frac{A P}{2}=\frac{A Q}{2}
$$




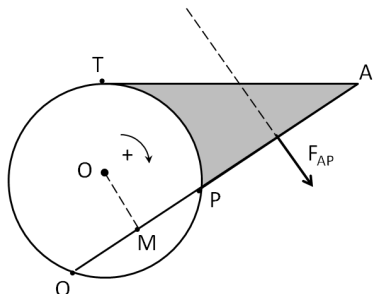

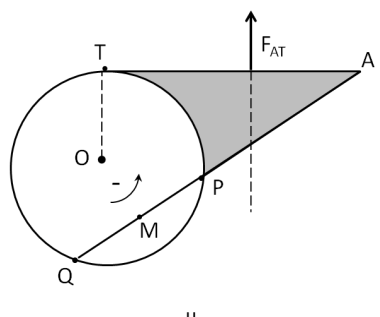

Figura 3: Distância do ponto $O$ à linha de ação das forças de pressão.

Aplicado-se agora as equações de equilíbrio dos torques $M_{O}$, temos que:

$$
\begin{gathered}
\sum M_{O}=0, \\
\overline{A T} \cdot \frac{\overline{A T}}{2}-\overline{A P} \cdot \frac{\overline{A Q}}{2}=0 \\
\overline{A T}^{2}=\overline{A P} \cdot \overline{A Q}
\end{gathered}
$$

Com as considerações dos conceitos físicos chegamos então a um resultado puramente geométrico.

Caso tivessemos atribuído uma pressão $p$ ou $h$ genéricas no início do problema, elas seriam simplificadas aqui neste ponto, ao se igualar o somatório dos torques $M_{O}$ a zero.

Em muitos problemas de geometria introduzimos "traçados auxiliares" ou "linhas de construção auxiliares" de forma a conduzir mais facilmente à resolução dos mesmos.

O que mostramos aqui, corresponde ao artifício de se introduzir uma "pressão auxiliar" conforme Figura 3, estudando assim as forças dela decorrentes e suas condições físicas de equilíbrio, que podem conduzir fisicamente da mesma forma que as linhas de construção dos problemas geométricos.

O mesmo raciocínio pode ser usado para as propriedades da potência de um ponto interior à circunferência.

A Figura 4 mostra a região ABD interna à circunferência, hermeticamente fechada, de forma a aprisionar um gás sob pressão. As resultantes das forças de pressão atuam sobre cada face deste recipiente sobre o centro geométrico destas e numericamente igual ao comprimento da respectiva face sobre a qual atuam. Esta condição não se aplica à face curvada $\widehat{\mathrm{AD}}$, mas tomando-se o ponto $O$, centro da circunferência, como referência para o cálculo de momentos, as forças de pressão sobre AD não causam tendências de giro em relação a este ponto, simplificando as contas.

Definimos assim as forças $F_{A B}$ e $F_{B D}$, as quais atuam sobre os pontos $K$ e $N$, pontos médios dos segmentos $\overline{A B}$ e $\overline{D B}$, respectivamente. Consideramos $L$ e $M$ como os pontos médios das cordas $\overline{A C}$ e $\overline{D E}$ da circunferência de cento $O$ e, os segmentos $\overline{O L}$ e $\overline{O M}$ paralelos às linhas de ação das forças de pressão $F_{A B}$ e $F_{B D}$. A distância $K L$ é dada por:

$$
K L=A L-A K=\frac{A C}{2}-\frac{A B}{2}=\frac{B C}{2}
$$




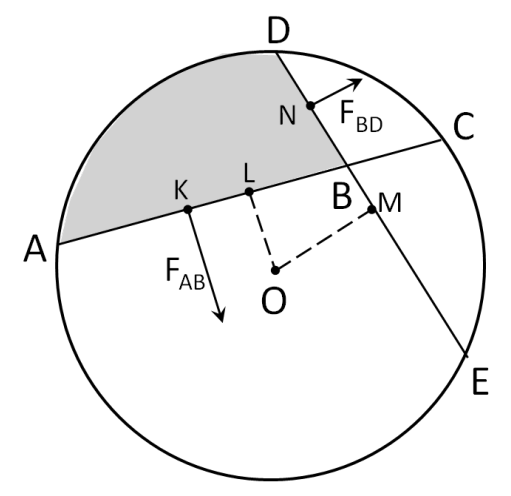

Figura 4: Demonstraçao física para a potência de um ponto interno.

Da mesma forma, a distância $M N$ é dada por:

$$
M N=D M-D N=\frac{D E}{2}-\frac{D B}{2}=\frac{B E}{2}
$$

Como cada uma destas forças causam uma tendência de giro no ponto $O$ em sentidos diferentes, seus momentos se igualam, e assim:

$$
\begin{gathered}
F_{A B} \cdot K L=F_{B D} \cdot M N \\
A B \cdot \frac{B C}{2}=B D \cdot \frac{B E}{2} \\
A B \cdot B C=B D \cdot B E
\end{gathered}
$$

o que demonstra o resultado esperado, como em (1).

Para complementar a exploração de problemas envolvendo a potência de um ponto podemos levar os estudantes a utilizarem o software livre de geometria dinâmica Geogebra, simulando diversas configurações e posições relativas de um ponto em relação a uma circunferência, checando as respectivas medidas e confirmando os resultados, o que fixará mais ainda o conteúdo.

Salientamos que para a compreensão da prova matemática exploramos os conhecimentos básicos de semelhança de triângulos e de arco capaz, e para a demonstração física retomamos os conceitos de pressão, força distribuida e força resultante, direção de atuação, bem como o conceito de torque e as condições para que uma força cause ou não torque em um determinado ponto.

\section{Conclusões}

O conteúdo de potência de um ponto em relação a uma circunferência ou problemas relacionados é apresentado geralmente em livros do 9o ano do Ensino Fundamental, período em que os alunos tiveram contato apenas com os primeiros conhecimentos de Física dentro da disciplina de Ciências. Esta proposta de aplicações da Física para o estudo da potência de um ponto não necessita ser vista como um primeiro contato com o tema no Ensino Fundamental, mas sim como um aprofundamento no Ensino Médio. Uma retomada de teoremas já conhecidos, desenvolvido com este novo olhar, é plenamente recomendável, pois reforça os resultados e revela aos estudantes que existem diversas 
formas de demonstração, como em [3], em que usamos ideias da Física para o estudo de propriedades de triângulos e o teorema de Pitágoras foi abordado com tal enfoque.

Percebemos que a demonstração de resultados geométricos utilizando argumentos detalhados e postulados puramente físicos mobiliza conceitos e estruturas mentais diferentes, em vez de uma demonstração abstrata usando argumentos puramente matemáticos, viabilizando atividades interdisciplinares para estudantes do Ensino Básico.

Com isso promovemos maior liberdade de pensamento para os estudantes, e enfatizamos que não existe um único caminho que conduz a demonstrações ou resultados corretos mas, múltiplas visões podem ser levadas em conta para se expressar e obter resultados, de maneira que o raciocínio dedutivo e o incremento das argumentações são aspectos gerais podendo ser enfocados no processo de ensino aprendizagem com sucesso.

Finalmente, acreditamos que esta abordagem interdisciplinar também é adequada para turmas especiais de preparação para olimpíadas de Matemática, Física ou Astronomia.

\section{Referências}

[1] Balacheff, N. Processus de preuve et situations de validation. Educational Studies in Mathematics, v. 18, n. 2, p. 147-176, 1987.

[2] Brasil Base Nacional Comum Curricular (BNCC). Educação é a Base. MEC/CONSED/UNDIME, Brasília, 2017.

[3] Boyer, C. B. Historia da Matemática, Revista por Uta C. Merzbach, São Paulo, Editora Edgard Blucher LTDA. 1996.

[4] Eves, H. Introdução a historia da matematica, Editora da UNICAMP, São Paulo, 1997.

[5] Levi, M. The Mathematical Mechanic: using physical reasoning to solve problems. Princeton University Press. 2009.

[6] Machado, S. D. A. Aprendizagem em Matemática: Registros de Representação Semiótica. Ensino da Matemática em Debate, São Paulo, volume 2, n. 2, jan. 2016. ISSN 2358-4122.

[7] Nasser e L. Tinoco, L. A. A. Argumentação e provas no ensino de Matemática. 2 ed. UFRJ/Projeto Fundão. Rio de Janeiro. 2003.

[8] Rodella, Y. M. e Salvador, J. A. Proposta de interdisciplinaridade: A experimentação física como auxílio didático a demonstrações matemáticas. Proceeding Series of the Brazilian Society of Computational and Applied Mathematics, volume 6, noㅡ 1, 2018. ISSN: 23590793

[9] São Paulo. Governo do Estado de São Paulo; Currículo do Estado de São Paulo - Matemática e suas Tecnologias. 2011.

[10] Wagner, E. Potência de um ponto em relação a uma circunferência, Revista do Professor de Matemática, vol. 45, IMPA, Rio de Janeiro, 2001. 